\title{
On Enlarging Backward Reachable Sets via Zonotopic Set Membership
}

\author{
Dongkun Han, Albert Rizaldi, Ahmed El-Guindy, and Matthias Althoff
}

\begin{abstract}
While a number of efficient methods have been proposed for approximating backward reachable sets, no synthesis method via backward reachable sets has been developed for estimating and enlarging the region of attraction (RA). This paper shows how to use backward reachable sets to enlarge the estimate of the RA of linear discrete-time systems, by using an optimal static feedback controller. Two controller design methods are provided: the first method enlarges the estimate of the RA via invariant sets, whose existence is ensured by zonotope containment; the second method provides the optimal control input by using Lyapunov stability and quadratic stabilization. The backward reachable set is represented by zonotopes which give a good compromise between accuracy and efficiency. The effectiveness of both methods is illustrated by a numerical example.
\end{abstract}

\section{INTRODUCTION}

The region of attraction (RA) of a given equilibrium point is the set of initial points from which the trajectories of a system converge to the considered equilibrium point. The asymptotical stability of operating points can be directly established if the RA is known. Recently, the importance of estimating the RA has gained a lot of interest and its implementations spread quickly to areas such as chemical reaction assessment [1], clinical immunotherapy [2], and biological processes [3].

In order to estimate the RA, methods based on Lyapunov's theorem have proven to be an efficient way [4]-[9]. In [4], the exact RA can be obtained by solving a partial differential equation (Zubov equation), but this equation is generally difficult to solve, which gives rise to a number of approaches using the sublevel set of Lyapunov functions. The estimation problem can be transformed to solvable conditions, like Linear Matrix Inequalities (LMI) [5], Sum of Squares (SOS) programming [10], Bilinear Matrix Inequalities (BMI) [6], and generalized eigenvalue problems [9]. Meanwhile, various types of Lyapunov functions are used, like quadratic Lyapunov functions [5], polyhedral Lyapunov functions [8], polynomial Lyapunov functions [6], pointwise maximum Lyapunov functions [11], and rational polynomial Lyapunov functions [9].

Non-Lyapunov methods also exist for estimating the RA [12]-[14]. Among these approaches, the backward reachable sets approach provides the domain from which the trajectories of a system converge to some selected target set, thus making it useful for estimating the RA of a given

The authors are with the Department of Informatics, Technical University of Munich, 85748 Garching, Germany. E-mail: \{hand, rizaldi, elguindy, althoff\}@in.tum.de
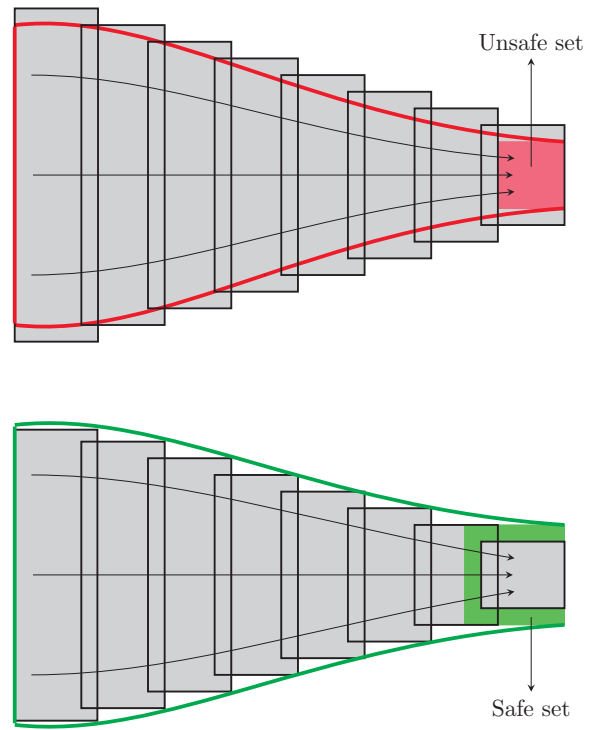

Fig. 1. Different strategies for approximating the backward reachable sets. The red box indicates the unsafe set whose backward reachable sets are outer-approximated by gray boxes; The green box indicates the safe set whose backward reachable sets are inner-approximated by gray boxes. The direction of system evolution is shown by some trajectories.

stable equilibrium point, if one selects the target set small enough near the equilibrium point. However, the backward reachable sets are usually computed with respect to an unsafe set, like aircraft collision avoidance and stability evaluation of underwater vehicles [13], [15], where outerapproximations of the exact backward reachable sets are computed. By contrast, for estimating the RA, one would like to ensure all the trajectories initializing in the estimate to be convergent to the equilibrium point. Thus, we compute the inner-approximation of the RA, which is shown in Fig. 1.

To compute the backward reachable set, Eulerian methods are mostly used, which require gridding of the state space, which means that they can not escape the curse of dimensionality. Lagrangian methods are an alternative since they avoid gridding of the state space, and they have been successfully used to compute forward reachable sets [16]-[18]. In [19], a dynamic programming technique is proposed and reachable sets are approximated by using ellipsoidal techniques. In [20], a Lagrangian method is proposed for computing the viability kernel via ellipsoidal representation. In [14], this method is extended by using polytopic and support vector representations. However, the problem of estimating and 
enlarging the RA is rarely considered via backward reachable sets.

Motivated by the work in [20], and by adopting the control purpose of our previous work [21], this paper proposes a Lagrangian method based on a zonotopic set representation. Different from the existing literature, this paper uses the backward reachable sets to estimate and enlarge the RA by designing an optimal controller. Specifically, the contributions of this paper are listed as follows:

- A method is proposed for checking the zonotope containment via Semi-Definite Programming (SDP). Based on this method, an optimal control input is obtained for enlarging the backward reachable set as the estimate of the RA by solving a BMI. (Section-III.A)

- By exploiting the Lyapunov stability theory, an optimal control input is computed for each step based on quadratic stabilization. The backward reachable set as the inner-approximation of the RA is maximized under the computed control input by solving a convex optimization problem. (Section-III.B)

\section{Preliminaries}

Notations: $\mathbb{N}, \mathbb{R}$ : natural and real number sets; $\mathbb{R}_{+}, \mathbb{R}_{++}$: nonnegative and positive real number sets; $0_{n}$ : origin of $\mathbb{R}^{n}$; $\mathbb{R}_{0}^{n}: \mathbb{R}^{n} \backslash\left\{0_{n}\right\} ; \mathbb{S}^{n \times n}, \mathbb{S}_{+}^{n \times n}, \mathbb{S}_{++}^{n \times n}:$ symmetric $n \times n$ matrices, symmetric positive and positive definite $n \times n$ matrices; $\|x\|_{2}$ : Euclidean norm $\left(l_{2}\right.$-norm) of vector $x ;\|x\|_{\infty}: l_{\infty}$-norm of vector $x ;[a ; b]$ : an interval $\{x \in \mathbb{R}: a \leq x \leq b\}$; $A^{T}$ : transpose of $A ; A>0(A \geq 0)$ : symmetric positive definite (semidefinite) matrix $A ; A \otimes B$ : Kronecker product of matrices $A$ and $B$; $\operatorname{trace}(A)$ : trace of matrix $A$; $\operatorname{diag}(v)$ : a square diagonal matrix with the elements of vector $v$ on the main diagonal; $\mathcal{A} \oplus \mathcal{B}$ : Minkowski sum of two sets $\mathcal{A}$ and $\mathcal{B}$, i.e.,

$$
\mathcal{A} \oplus \mathcal{B}=\{a+b: a \in \mathcal{A}, b \in \mathcal{B}\}
$$

$\mathrm{CH}(\mathcal{A}, \mathcal{B})$ : Convex hull of sets $\mathcal{A}$ and $\mathcal{B}$, i.e.,

$$
\mathrm{CH}(\mathcal{A}, \mathcal{B})=\{(1-\alpha) a+\alpha b: a \in \mathcal{A}, b \in \mathcal{B}, \alpha \in[0,1]\} ;
$$

In this section, we first introduce the definition and some basic properties of zonotopes. Then, we concisely show the main idea to obtain the stability region of an equilibrium point based on the computation of backward reachable sets. This section ends with a formal problem formulation.

\section{A. Zonotopic Sets}

A convex polytope $\mathcal{P} \subseteq \mathbb{R}^{n}$ is the convex hull of a group of vertices, i.e., $\mathrm{CH}\left(\mathrm{v}^{(1)}, \ldots, \mathrm{v}^{\left(n_{\mathrm{v}}\right)}\right):=$ $\left\{\sum_{i=1}^{n_{\mathrm{v}}} \alpha_{i} \mathrm{v}^{(i)}: \mathrm{v}^{(i)} \in \mathbb{R}^{n}, \alpha_{i} \in \mathbb{R}_{++}, \quad \sum_{i=1}^{n_{\mathrm{v}}} \alpha_{i}=1\right\}$. Besides the vertex representation (V-rep), $\mathcal{P}$ can also be expressed by halfspaces (H-rep): For $n_{h}$ halfspaces, $\mathcal{P}=$ $\left\{x \in \mathbb{R}^{n}: H \cdot x \leq d, H \in \mathbb{R}^{n_{h} \times n}, d \in \mathbb{R}^{n_{h}}\right\}[22]$.

Zonotopes are special convex polytopes with central symmetry. This symmetry allows the zonotopes to be expressed by the following two expressions: The infinity-norm representation (I-rep) (See [23], [24] and references therein):

$$
\left\{x \in \mathbb{R}^{n}:\|W(x-c)\|_{\infty} \leq 1\right\},
$$

where $W \in \mathbb{R}^{n_{w} \times n}$, and $c \in \mathbb{R}^{n}$ denotes the center. Another expression for zonotope is the generator representation (Grep):

$$
\mathcal{Z}(c, G)=\left\{x \in \mathbb{R}^{n}: x=c \oplus G \Xi\right\}
$$

where

$$
\Xi=\left\{\zeta \in \mathbb{R}^{m}:\|\zeta\|_{\infty} \leq 1\right\}
$$

$c \in \mathbb{R}^{n}$ denotes the center, $G \in \mathbb{R}^{n \times m}: G=\left[g_{1}, \ldots, g_{m}\right]$ denotes the generator matrix of the zonotope. We call $g_{i}$ a generator which is a column vector of matrix $G$, for all $i=1, \ldots, m$. This definition shows the fact that a zonotope is built by the Minkowski sum of a group of line segments $l_{i}=[-1 ; 1] \cdot g_{i}$, where $[-1 ; 1]$ is the unit interval [18], [22], [25]. The following example illustrates the construction of a zonotope.

Example 1: Consider a zonotope $\mathcal{Z}_{1}\left(c_{1}, G_{1}\right)$ where

$$
c_{1}=\left[\begin{array}{l}
1 \\
2
\end{array}\right], G_{1}=\left[\begin{array}{lll}
1 & 0 & 1 \\
1 & 1 & 0
\end{array}\right] \text {. }
$$

Let $g_{1}^{(i)}$ be the $i$-th column vector of $G_{1}$ and define the line segments $l_{i}=[-1 ; 1] \cdot g^{(i)}$, for $i=1,2,3$. Fig. 2 shows how the zonotope $\mathcal{Z}_{1}$ is constructed by a group of line segments.
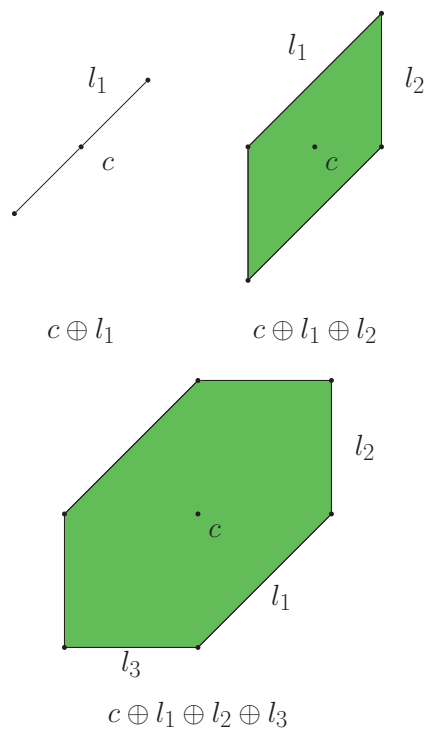

Fig. 2. Construction of zonotope step-by-step.

Another explanation of (2) is that a zonotope $\mathcal{Z}(c, G)$ is an affine image of a unit cube $\left\{\zeta \in \mathbb{R}^{m}:\|\zeta\|_{\infty} \leq 1\right\}$ under a linear mapping $G$ using $l_{\infty}$ norm, just as an ellipsoid $\mathcal{E}=\left\{x \in \mathbb{R}^{n}: x=c+R \zeta,\|\zeta\|_{2} \leq 1\right\}$ is an affine image of a unit ball $\left\{\zeta \in \mathbb{R}^{m}:\|\zeta\|_{2} \leq 1\right\}$ under a linear mapping $R$ using the $l_{2}$ norm. Note that for a single ellipsoidal representation, the complexity is fixed for a selected $n$, while the complexity of representations using convex polytopes and zonotopes can be arbitrarily high [26]. In other words, by using polytopes and zonotopes, an increasing accurate 
representation can be obtained according to a higher userdefined complexity. The order $o_{c}=\frac{m}{n}$ shows the complexity of zonotopic representations.

The applicability of a set representation depends largely on whether required operations satisfy the closure property. The following two basic set operations are considered in this paper:

- Linear mapping: $B \mathcal{A}:=\{B a: a \in \mathcal{A}\}$;

- Minkowski sum: $\mathcal{A} \oplus \mathcal{B}:=\{a+b: a \in \mathcal{A}, b \in \mathcal{B}\}$, where $B \in \mathbb{R}^{n_{b} \times n}$, and $\mathcal{A}, \mathcal{B} \subseteq \mathbb{R}^{n}$. The closure of these set operations for different set representations is shown in Tab. I.

TABLE I

THE CLOSURE OF SET OPERATIONS FOR DIFFERENT SET REPRESENTATIONS.

\begin{tabular}{lccc}
\hline & Ellipsoid & Zonotope & Polytope \\
\hline Linear mapping & closed & closed & closed \\
Minkowski sum & not closed & closed & closed \\
\hline
\end{tabular}

Zonotopes are preferred not only because of the closure for the above two operations, but the computations of these operations are also efficient via G-rep [18], [27]. For zonotopes $\mathcal{Z}_{1}\left(c_{1}, G_{1}\right), \mathcal{Z}_{2}\left(c_{2}, G_{2}\right) \subseteq \mathbb{R}^{n}$ and matrices $B \in \mathbb{R}^{n_{b} \times n}$, $G_{1} \in \mathbb{R}^{n \times m_{1}}, G_{2} \in \mathbb{R}^{n \times m_{2}}$ the number of operation is shown in Tab. II.

TABLE II

THE NUMBER OF BINARY OPERATIONS $n_{p}$ FOR ZONOTOPIC CALCULUS.

\begin{tabular}{cc}
\hline Set operation & $n_{p}$ \\
\hline $\mathcal{Z}_{1}\left(c_{1}, G_{1}\right) \oplus \mathcal{Z}_{2}\left(c_{2}, G_{2}\right)=\mathcal{Z}\left(c_{1}+c_{2},\left[G_{1} G_{2}\right]\right)$ & $n$ \\
$B \mathcal{Z}_{1}=Z\left(B \cdot c_{1}, B \cdot G_{1}\right)$ & $2 n_{b} n\left(m_{1}+1\right)$ \\
\hline
\end{tabular}

Though convex polytopes are closed for the operations of linear mapping and Minkowski sum, the complexity of both operations is exponential via H-rep in the worst case scenario [28]. By contrast, as shown in Tab. II, the computation is more efficient via G-rep and the complexity increases moderately for both operations in Tab. I. In addition, there exist some effective order reduction methods to control the complexity of zonotopes. Both outer- and inner-approximations with reduced order are proposed for reachability analysis, making the zonotopic set membership a balanced representation between accuracy and complexity.

\section{B. Problem Formulation}

Consider the nonlinear time-varying discrete-time system:

$$
x(k+1)=f(x(k), u(x(k)), k),
$$

where $x(k) \in \mathbb{R}^{n}$ is the state vector at the sampling time $k, x(0) \in \mathbb{R}^{n}$ is the initial state, $u(\cdot): \mathbb{R}^{n} \rightarrow \mathcal{U} \subseteq \mathbb{R}^{n_{u}}$ is the measurable static feedback input of system (5), $f$ : $\mathbb{R}^{n} \times \mathcal{U} \times \mathbb{N} \rightarrow \mathbb{R}^{n}$ is a linearizable nonlinear function satisfying the local Lipschitz condition. Assume that system (5) has a locally stable equilibrium point and without loss of generality, the origin is set to be the equilibrium point of interest. From now on, the arguments $k$ and $x$ will be omitted whenever possible for brevity of notation.

The backward reachable set is a set that can be steered into a given terminal set (or called target set) under a series of control inputs. Specifically, given a terminal time $k_{\mathrm{t}}$ and a terminal set $\mathcal{T}$, the backward reachable set $\Psi(k, \mathcal{T})$ at time step $k$ is defined as follows.

Definition 1: (Backward Controlled Reachable Set) The backward reachable set $\Psi(k, \mathcal{T})$ of system (5) at time step $k<k_{\mathrm{t}}$ is the set of states $x(k)$, for any of which there exists a series of control inputs $u(x(k)), \ldots, u\left(x\left(k_{\mathrm{t}}-1\right)\right)$ such that the system is driven from $x(k)$ to $x\left(k_{\mathrm{t}}\right) \in \mathcal{T}$, i.e.,

$$
\Psi(k, \mathcal{T})=\left\{\begin{array}{lr}
\left\{x(k) \in \mathbb{R}^{n}: \exists u(\tau) \in \mathcal{U},\right. \\
x(\tau+1)=f(x(\tau), u(x(\tau)), \tau), x\left(k_{\mathrm{t}}\right) \in \mathcal{T}, \\
\left.\forall \tau=k, \ldots,\left(k_{\mathrm{t}}-1\right)\right\}, & k<k_{\mathrm{t}} \\
\mathcal{T}, & k=k_{\mathrm{t}} .
\end{array}\right.
$$

Definition 2: The RA of system (5) is the set of initial states for which the system asymptotically converges to the origin, i.e.,

$$
\mathcal{R}=\left\{x(0) \in \mathbb{R}^{n}: \lim _{\tau \rightarrow+\infty} \chi(\tau ; x(0), u(\tau))=0_{n}\right\}
$$

where $\chi$ is the solution of system (5).

Based on Definition 1, the following result shows how backward reachable sets can be used to estimate the RA.

Lemma 1: Given a terminal set $\mathcal{T} \subseteq \mathcal{R}$, if the backward reachable set at time step $k$ always contains the backward reachable set at the next time step, i.e.,

$$
\Psi(\tau+1, \mathcal{T}) \subseteq \Psi(\tau, \mathcal{T}), \forall \tau=k, \ldots,\left(k_{\mathrm{t}}-1\right),
$$

then, the backward reachable set at time step $k$ is an estimate of the RA, i.e., $\Psi(k, \mathcal{T}) \subseteq \mathcal{R}$.

Proof: Since $\mathcal{T} \subseteq \mathcal{R}$, based on Definition 1, one has that there exists a $u\left(k_{\mathrm{t}}-1\right)$ steering $\Psi\left(k_{\mathrm{t}}-1, \mathcal{T}\right)$ to $\mathcal{T}$. Taking Definition 2 into account, one has that $\Psi\left(k_{\mathrm{t}}, \mathcal{T}\right)=\mathcal{T} \subseteq$ $\Psi\left(k_{\mathrm{t}}-1, \mathcal{T}\right) \subseteq \mathcal{R}$. Analogously, it yields that

$$
\mathcal{T} \subseteq \Psi(\tau+1, \mathcal{T}) \subseteq \Psi(\tau, \mathcal{T}) \subseteq \mathcal{R}, \forall \tau=k, \ldots,\left(k_{\mathrm{t}}-1\right)
$$

which ends this proof.

Let us propose the main problem we are concerned with: Find an optimal static feedback controller $u(x(k))$ at time step $k$ such that the estimate of $\mathcal{R}$ is maximized with respect to a measure $\rho(\Psi(k, \mathcal{T}))$, i.e., solving

$$
\begin{aligned}
& \mu=\sup ^{u} \rho(\Psi(k, \mathcal{T})) \\
& \text { s.t. }\left\{\begin{array}{l}
\Psi(\tau+1, \mathcal{T}) \subseteq \Psi(\tau, \mathcal{T}), \forall \tau=k, \ldots,\left(k_{\mathrm{t}}-1\right), \\
\Psi\left(k_{\mathrm{t}}\right)=\mathcal{T}
\end{array}\right.
\end{aligned}
$$




\section{MAIN RESUlts}

In this section, we propose two methods to estimate the RA. The first one provides a control strategy based on the zonotopic containment. The other one searches for the optimal controller via quadratic Lyapunov stability. Algorithms based on solving tractable optimization problems are proposed for both methods.

\section{A. Enlargement based on Zonotopic Containment}

We consider a discrete-time linear system with a static feedback controller,

$$
x(k+1)=A x(k)+B u(k), u(k)=F(k) x(k),
$$

with $A \in \mathbb{R}^{n \times n}, B \in \mathbb{R}^{n \times n_{u}}$ and $F \in \mathbb{R}^{n_{u} \times n}$. Note that we use the linear system (9) to illustrate the main idea of our approach, which can be extended to the linearizable nonlinear systems as in [17].

Remark 1: For this model, we assume that

- The pair $(A, B)$ of system (9) is controllable, from which one has that $A(k)+B(k) F(k)$ is invertible.

- the control input is bounded and $F(k)$ is constrained by an arbitrary hyper-rectangle:

$$
\begin{aligned}
\mathcal{F}= & \left\{F: F_{i j} \in\left[\underline{F}_{i j}, \bar{F}_{i j}\right],\right. \\
& \left.i=1, \ldots, n_{u}, j=1, \ldots, n\right\} .
\end{aligned}
$$

For system (9), we use the zonotopic set membership to represent the backward reachable sets. Specifically, let the terminal set $\mathcal{T}$ be defined by $\mathcal{T}=c_{\mathrm{t}} \oplus G_{\mathrm{t}} \Xi$. From (9), we have

$$
\Psi(k, \mathcal{T})=(A+B F(k))^{-1} \Psi(k+1, \mathcal{T}) .
$$

Then, by iteratively using (11), the backward reachable set at time step $k$ can be computed as

$$
\Psi(k, \mathcal{T})=c_{k} \oplus G_{k} \Xi
$$

with

$$
\begin{aligned}
& \Xi=\left\{\zeta \in \mathbb{R}^{n}:\|\zeta\|_{\infty} \leq 1\right\}, \\
& c_{k}=\Delta(k) c_{\mathrm{t}}, G_{k}=\Delta(k) G_{\mathrm{t}}, \\
& \Delta(k)=\prod_{\tau=k}^{k_{\mathrm{t}}-1}(A+B F(\tau))^{-1} .
\end{aligned}
$$

Before we propose the main result, we present the following lemma.

Lemma 2 ([29]): For a finite dimensional vector space, the $l_{2}$ norm and $l_{\infty}$ norm satisfy the following inequality

$$
\|x\|_{\infty} \leq\|x\|_{2} \leq \sqrt{n}\|x\|_{\infty} .
$$

The ellipsoidal calculus is well developed and is related to convex optimization [30], where the ellipsoid containment can be established efficiently by an LMI condition via the S-procedure [31]. However, the problem of zonotope containment is rarely considered. The following result gives a solution for this problem via solving an LMI.

Lemma 3 (Zonotope Containment): Given zonotopes $\mathcal{Z}_{1}=\left\{x_{1} \in \mathbb{R}^{n}:\left\|H_{1}\left(x_{1}-c_{1}\right)\right\|_{\infty} \leq 1\right\}$ and
$\mathcal{Z}_{2}=\left\{x_{2} \in \mathbb{R}^{n}:\left\|H_{2}\left(x_{2}-c_{2}\right)\right\|_{\infty} \leq 1\right\}$ with $H_{1} \in \mathbb{R}^{m_{1} \times n}$ and $H_{2} \in \mathbb{R}^{m_{2} \times n}, \mathcal{Z}_{1} \subseteq \mathcal{Z}_{2}$ if there exists a positive scalar $0<\lambda<1$ and the following LMI holds:

$$
Q=\left[\begin{array}{ccc}
Q_{11} & Q_{12} & Q_{13} \\
* & -\lambda^{2} \bar{H}_{1}^{T} \bar{H}_{1} & \lambda \bar{H}_{1}^{T} \bar{H}_{2} \\
* & * & -\bar{H}_{2}^{T} \bar{H}_{2}
\end{array}\right] \geq 0,
$$

where $*$ denotes the terms for the symmetry, $\bar{H}_{1} \in \mathbb{R}^{m_{3} \times n}$ and $\bar{H}_{2} \in \mathbb{R}^{m_{3} \times n}$ are extended matrices of $H_{1}$ and $H_{2}$ with $m_{3}=\max \left\{m_{1}, m_{2}\right\}$ and the expressions

$$
\bar{H}_{1}=\left[\begin{array}{c}
H_{1} \\
0_{\left(m_{3}-m_{1}\right) \times n}
\end{array}\right], \bar{H}_{2}=\left[\begin{array}{c}
H_{2} \\
0_{\left(m_{3}-m_{2}\right) \times n}
\end{array}\right],
$$

and

$$
\begin{aligned}
& Q_{11}=(1-\lambda)^{2}-\left(\bar{H}_{2} c_{2}-\lambda \bar{H}_{1} c_{1}\right)^{T}\left(\bar{H}_{2} c_{2}-\lambda \bar{H}_{1} c_{1}\right), \\
& Q_{12}=\lambda\left(\lambda \bar{H}_{1} c_{1}-\bar{H}_{2} c_{2}\right)^{T} \bar{H}_{1}, \\
& Q_{13}=\left(\lambda \bar{H}_{1} c_{1}-\bar{H}_{2} c_{2}\right)^{T} \bar{H}_{2} .
\end{aligned}
$$

Proof: $\mathcal{Z}_{1} \subseteq \mathcal{Z}_{2}$ holds if there exists a $0<\lambda<1$ such that

$$
1-\left\|H_{2}\left(x-c_{2}\right)\right\|_{\infty}-\lambda\left(1-\left\|H_{1}\left(x-c_{1}\right)\right\|_{\infty}\right) \geq 0,
$$

i.e., $\left\|H_{1}\left(x-c_{1}\right)\right\|_{\infty} \leq 1 \Rightarrow\left\|H_{2}\left(x-c_{2}\right)\right\|_{\infty} \leq 1$. One can rewrite (16) as

$$
\left\|H_{2}\left(x-c_{2}\right)\right\|_{\infty}-\lambda\left\|H_{1}\left(x-c_{1}\right)\right\|_{\infty} \leq 1-\lambda,
$$

which is equivalent to the following inequality by replacing $H_{1}$ and $H_{2}$ with $\bar{H}_{1}$ and $\bar{H}_{2}$ :

$$
\left\|\bar{H}_{2}\left(x-c_{2}\right)\right\|_{\infty}-\lambda\left\|\bar{H}_{1}\left(x-c_{1}\right)\right\|_{\infty} \leq 1-\lambda .
$$

This condition holds if

$$
\left\|\bar{H}_{2}\left(x-c_{2}\right)-\lambda \bar{H}_{1}\left(x-c_{1}\right)\right\|_{\infty} \leq 1-\lambda
$$

based on the properties of the $l_{\infty}$ norm: $\alpha\|A\|_{\infty}=\|\alpha A\|_{\infty}$ for any $\alpha>0$ and $\|A-B\|_{\infty} \geq\|A\|_{\infty}-\|B\|_{\infty}$. From Lemma 2, one has that (19) holds if

$$
\left\|\bar{H}_{2}\left(x-c_{2}\right)-\lambda \bar{H}_{1}\left(x-c_{1}\right)\right\|_{2} \leq 1-\lambda,
$$

which yields that $\left(\bar{H}_{2}\left(x-c_{2}\right)-\lambda \bar{H}_{1}\left(x-c_{1}\right)\right)^{T}\left(\bar{H}_{2}\left(x-c_{2}\right)-\right.$ $\left.\lambda \bar{H}_{1}\left(x-c_{1}\right)\right) \leq(1-\lambda)^{2}$. This is equivalent to

$$
\left[\begin{array}{l}
1 \\
x \\
x
\end{array}\right]^{T} Q\left[\begin{array}{l}
1 \\
x \\
x
\end{array}\right] \geq 0
$$

which ends the proof.

Remark 2: Let us observe that the expression of zonotopes in Lemma 3 is I-rep rather than G-rep. Thus, a transformation from G-rep of zonotopes to I-rep is needed. Consider the zonotope $\mathcal{Z}(c, G)=\left\{x \in \mathbb{R}^{n}: x=c+G \zeta,\|\zeta\|_{\infty} \leq 1\right\}$, let $\varepsilon \in \mathbb{R}^{n}$ and $\varepsilon=c+G \zeta$, one has $\zeta=G_{\text {left }}^{-1}(\varepsilon-c)$, where $G_{\text {left }}^{-1}$ is the left inverse of matrix $G$. Since $\|\zeta\|_{\infty} \leq 1$, a expression is obtained that $\mathcal{Z}=\left\{\varepsilon \in \mathbb{R}^{n}:\left\|G_{\text {left }}^{-1}(\varepsilon-c)\right\|_{\infty} \leq\right.$ $1\}$. Note that $G_{\text {left }}^{-1} G=I_{m}$ and $G_{\text {left }}^{-1}$ exists only if $G$ has full column rank. Thus, regarding a generator matrix $G \in \mathbb{R}^{n \times m}$, 1) for the case of parallelotopes, one has that $\operatorname{rank}(G)=m$ [32], it yields that $\left(G^{T} G\right)$ is invertible and $\left(G^{T} G\right)^{-1} G^{T}$ is a left inverse of $G$; 2 ) for general zonotopes with G-rep, one 
needs to first change the form to H-rep (by using the method in [22]), from which I-rep can be easily obtained.

In addition, it is worth noting that condition (15) is only a sufficient condition. It is still an open question to find a necessary and sufficient LMI condition for zonotope containment.

We give an example to illustrate the above result.

Example 2: Consider $\mathcal{Z}_{1}\left(c_{1}, G_{1}\right)$ and $\mathcal{Z}_{2}\left(c_{2}, G_{2}\right)$ with

$$
\begin{aligned}
& c_{1}=\left[\begin{array}{c}
0 \\
10
\end{array}\right], G_{1}=\left[\begin{array}{cc}
500 & 0 \\
0 & 500
\end{array}\right], \\
& c_{2}=\left[\begin{array}{c}
10 \\
0
\end{array}\right], G_{2}=\left[\begin{array}{cc}
900 & 300 \\
300 & 900
\end{array}\right] .
\end{aligned}
$$

For this case, one has $H_{1}=G_{1}^{-1}$ and $H_{2}=G_{2}^{-1}$. From (15), it yields that $Q \geq 0$. Thus, $\mathcal{Z}_{1} \subseteq \mathcal{Z}_{2}$, which is shown in Fig. 3.

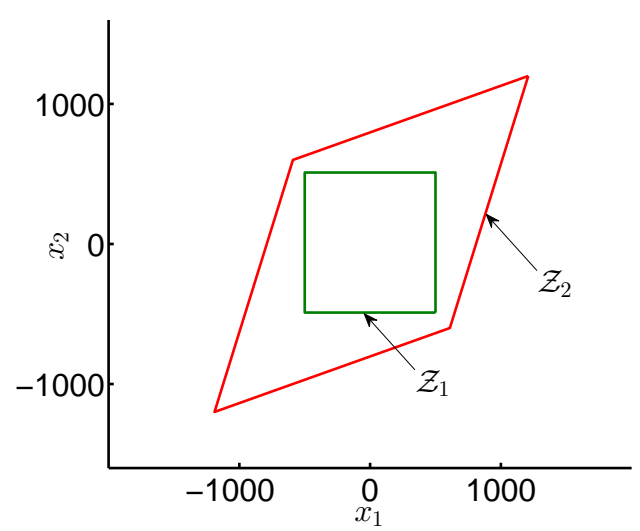

Fig. 3. Example 2 for illustration of zonotope containment: The green shape indicates $\mathcal{Z}_{1}$ and the red one indicates $\mathcal{Z}_{2}$.

Now, we propose a new method based on zonotope containment.

Theorem 1: For system (9), assume that $\mathcal{T}=\mathcal{Z}\left(c_{\mathrm{t}}, G_{\mathrm{t}}\right)$ and matrix $G_{\mathrm{t}}$ has full column rank. If there exist a positive scalar $0<\lambda<1$ and a series of control inputs $u(k)=$ $F(k) x(k)$ with $F(k)$ (denoted as $F_{k}$ to the end) being the optimal value of the following optimization:

$$
\begin{aligned}
& \rho=\min _{F_{k}} \operatorname{trace}\left(S\left(F_{k}\right)\right) \\
& Q_{k}=\left[\begin{array}{ccc}
Q_{k 11} & Q_{k 12} & Q_{k 13} \\
* & -\lambda^{2} H_{k+1}^{T} H_{k+1} & \lambda H_{k+1}^{T} H_{k} \\
* & * & -H_{k}^{T} H_{k}
\end{array}\right] \geq 0 \\
& \operatorname{diag}\left(F_{k 11}-\underline{F}_{11}, \bar{F}_{11}-F_{k 11}, \ldots,\right. \\
& \left.\quad F_{k n_{u} n}-\underline{F}_{n_{u} n}, \bar{F}_{k n_{u} n}-F_{n_{u} n}\right) \geq 0
\end{aligned}
$$

where

$$
\begin{aligned}
S\left(F_{k}\right)= & \left(A+B F_{k}\right)^{T}\left(A+B F_{k}\right) \\
Q_{k 11}= & (1-\lambda)^{2}-\left(H_{k} c_{k}-\lambda H_{k+1} c_{k+1}\right)^{T} \\
& \cdot\left(H_{k} c_{k}-\lambda H_{k+1} c_{k+1}\right) \\
Q_{k 12}= & \lambda\left(\lambda H_{k+1} c_{k+1}-H_{k} c_{k}\right)^{T} H_{k+1}, \\
Q_{k 13}= & \left(\lambda H_{k+1} c_{k+1}-H_{k} c_{k}\right)^{T} H_{k}
\end{aligned}
$$

and $H_{k}, H_{k+1}$ are the left inverses of generator matrices $G_{k}, G_{k+1}$, respectively, then, $\Psi(k, \mathcal{T})=\mathcal{Z}\left(c_{k}, G_{k}\right)$ is an inner-approximation of $\mathcal{R}$.

Proof: Since $G_{\mathrm{t}}$ has full column rank, according to the controllability of system (9) and (13), one has that $G_{k}$ is a full-column-rank matrix. Thus, for any $k \in \mathbb{N}, G_{k}$ has a left inverse $H_{k}=G_{k-\text { left }}$. Since $G_{k+1}=\left(A+B F_{k}\right) G_{k}$ and $G_{k-\text { left }} G_{k}=I_{m}$, one has that

$$
H_{k}=H_{k+1}\left(A+B F_{k}\right)
$$

where $H_{k+1}=\left(G_{k+1}^{T} G_{k+1}\right)^{-1} G_{k+1}^{T}$. If there exist a positive scalar $0<\lambda<1$ and control inputs $F_{k}$ such that $Q_{k} \geq 0$, from Lemma 3 , one can conclude $\mathcal{Z}\left(c_{k}, G_{k}\right) \subseteq$ $\mathcal{Z}\left(c_{k-1}, G_{k-1}\right)$.

By taking $\Psi(k, \mathcal{T})=\mathcal{Z}\left(c_{k}, G_{k}\right)$ into account, one has that the backward reachable set at time step $k$ always contains the backward reachable set at time step $k+1$, i.e.,

$$
\Psi(\tau+1, \mathcal{T}) \subseteq \Psi(\tau, \mathcal{T}), \forall \tau=k, \ldots,\left(k_{\mathrm{t}}-1\right) .
$$

Thus, from Lemma 1 , it yields that $\Psi(k, \mathcal{T}) \subseteq \mathcal{R}$.

Finally, let us choose the Frobenius norm of the generator matrix as a measure of zonotopes used in [25]. In order to enlarge the backward reachable set $\Psi(k, \mathcal{T})$, one can maximize the Frobenius norm of the corresponding generator matrix, i.e., $\rho(\Psi(k+1, \mathcal{T}))=\left\|G_{k}\right\|_{F}^{2}=$ $\operatorname{trace}\left(\left(G_{k}\right)^{T}\left(G_{k}\right)\right)$, which is equivalent to maximizing $\operatorname{trace}\left(\left(\left(A+B F_{k}\right)^{-1}\left(G_{k+1}\right)\right)^{T}\left(\left(A+B F_{k}\right)^{-1} G_{k+1}\right)\right)$. To do this, considering the facts that the eigenvalues of $(((A+$ $\left.\left.\left.B F_{k}\right)^{-1}\right)^{T}\left(\left(A+B F_{k}\right)^{-1}\right)\right)$ are positive and are reciprocals of corresponding eigenvalues of $\left(A+B F_{k}\right)^{T}\left(A+B F_{k}\right)$, one can maximize trace $\left(\left(A+B F_{k}\right)^{-1}\left(G_{k+1}\right)\right)^{T}((A+$ $\left.\left.\left.B F_{k}\right)^{-1} G_{k+1}\right)\right)$ by approximately minimizing $\operatorname{trace}((A+$ $\left.\left.B F_{k}\right)^{T}\left(A+B F_{k}\right)\right)$, which ends this proof.

Remark 3: For this result, it is worth noting that: Constraints in (22) is a Bilinear Matrix Inequality (BMI) [31]. One strategy to solve this problem is by replacing the quadratic terms by new variables and outer-approximating the bounds of these new variables [33]. Another strategy is the alternating SDP method (Gauss-Seidel method), which iteratively solves SDP via switching variables to avoid quadratic terms [34].

\section{B. Enlargement based on Quadratic Stabilization}

Besides the zonotope containment method, Lyapunov stability methods have been proven to be efficient for estimating the RA. In this subsection, we propose a method to enlarge the backward reachable set based on quadratic stabilization.

Let us consider the system (9) where the assumptions in Remark 2 are satisfied. The main idea of this method is to compute a feasible feedback control matrix $F$ to ensure the quadratic stability, i.e., the poles of the matrix $A+B F$ are kept in a region of the unit disk. In this way, the backward reachable sets are guaranteed to be the estimate of the RA.

Lemma 4: The system (9) is $\gamma$-stable (i.e. stable with converging rate $\gamma$ ) if there exist matrices $W \in \mathbb{S}_{++}^{n \times n}$ and 
$Y \in \mathbb{R}^{n_{u} \times n}$ such that

$$
\left[\begin{array}{cc}
\gamma W & W A^{T}+Y^{T} B^{T} \\
A W+B Y & W
\end{array}\right]>0,
$$

then, $F=Y W^{-1}$ ensures the quadratic $\gamma$-stability of system (9).

Proof: By calculating the Schur complement of (24), one has

$$
\gamma W-\left(W A^{T}+Y^{T} B^{T}\right) W^{-1}(A W+B Y)>0,
$$

which yields that for any $k \geq 0$ and $x(k) \neq 0$,

$$
\begin{aligned}
& \gamma x(k)^{T} W x(k)> \\
& x(k)^{T}\left(W A^{T}+Y^{T} B^{T}\right) W^{-1}(A W+B Y) x(k), \\
& \gamma x(k)^{T} W x(k)>x(k+1)^{T} W x(k+1) .
\end{aligned}
$$

Thus, consider a quadratic Lyapunov function $V(k)=$ $x(k)^{T} W x(k)$, one has $V(k+1)<\gamma V(k)$, which completes this proof.

Remark 4: Regarding Lemma 4, for the case of $\gamma=1$, (24) reduces to the Schur stability of system (9).

Lemma 5: Consider a series of backward reachable sets $\Psi\left(k_{0}, \mathcal{T}\right), \Psi\left(k_{0}+1, \mathcal{T}\right), \ldots, \Psi\left(k_{\mathrm{t}}, \mathcal{T}\right)$, provided that there is a $\bar{k}$ with $\Psi(\bar{k}, \mathcal{T}) \subseteq \mathcal{R}$, then, for any $k_{0} \leq k<\bar{k}, k \in \mathbb{N}$, there exists a group of control inputs $u(\tau), \tau=k, \ldots, \bar{k}-1$ such that $\Psi(k, \mathcal{T}) \subseteq \mathcal{R}$.

Proof: Let us observe from Definition 1 that the backward reachable set has the following semigroup property: For all $k_{0} \leq k \leq k_{\mathrm{t}}$,

$$
\Psi\left(k_{0}, \mathcal{T}\right)=\Psi\left(k_{0}, \Psi(k, \mathcal{T})\right)
$$

under the control inputs $u(\tau), \tau=k_{0}, \ldots, k_{\mathrm{t}}-1$. Thus, there exists a group of control inputs $\bar{u}(\tau), \tau=k, \ldots, \bar{k}-1$ such that $\Psi(k, \mathcal{T})$ can be steered into $\Psi(\bar{k}, \mathcal{T})$, and there also exists a group of control inputs $\bar{u}(\tau), \tau=\bar{k}, \ldots, k_{\mathrm{t}}-1$ such that $\Psi(\bar{k}, \mathcal{T})$ can be steered into $\Psi\left(k_{\mathrm{t}}, \mathcal{T}\right)$. By taking Definition 2 into account, if $\Psi(\bar{k}, \mathcal{T}) \subseteq \mathcal{R}$ and $\Psi\left(k_{\mathrm{t}}, \mathcal{T}\right)=$ $\mathcal{T} \subseteq \mathcal{R}$, it yields that under the control inputs $\bar{u}(\tau)$, that $\tau=k, \ldots, k_{\mathrm{t}}-1, \Psi(k, \mathcal{T}) \subseteq \mathcal{R}$, which completes this proof.

Theorem 2: Consider the system (9) satisfying the assumptions in Remark 2, if there exist matrices $W \in \mathbb{S}_{++}^{n \times n}$, $Y \in \mathbb{R}^{n_{u} \times n}$, a positive scalar $\gamma \in \mathbb{R}_{++}$and criteria $\rho$ such that

$$
\begin{aligned}
& \rho=\min _{W, Y} \operatorname{trace}(R(W, Y)) \\
& {\left[\begin{array}{cc}
\gamma W & W A^{T}+Y^{T} B^{T} \\
A W+B Y & W
\end{array}\right]>0,} \\
& {\left[\begin{array}{cc}
1 & v_{k}^{(r)^{T}} \\
v_{k}^{(r)} & W
\end{array}\right]>0, \forall r=1, \ldots, n_{v},} \\
& \operatorname{diag}\left(Y_{11}-\underline{Y}_{11}, \bar{Y}_{11}-Y_{11}, \ldots,\right. \\
& \left.Y_{n_{u} n}-\underline{Y}_{n_{u} n}, \bar{Y}_{n_{u} n}-Y_{n_{u} n}\right) \geq 0,
\end{aligned}
$$

where

$$
\begin{aligned}
& R(W, Y)=(A W+B Y)^{T}(A W+B Y), \\
& \underline{y}_{i j}=\sum_{k=1}^{n} \min \left(\underline{F}_{i k} W_{k i}, \bar{F}_{i k} W_{k i}\right), \\
& \bar{y}_{i j}=\sum_{k=1}^{n} \max \left(\underline{F}_{i k} W_{k i}, \bar{F}_{i k} W_{k i}\right),
\end{aligned}
$$

for all $i=1, \ldots, n_{u}, j=1, \ldots, n$, and $v_{k}^{(r)}$ is the vertex of zonotope $\Psi(k)$, then, the backward reachable set satisfies $\Psi(k-1) \subseteq \mathcal{R}$.

Proof: From Lemma 4, the first inequality of (26) ensures that there exist matrices $W \in \mathbb{S}_{++}^{n \times n}, Y \in \mathbb{R}^{n_{u} \times n}$ and a positive scalar $\gamma \in \mathbb{R}_{++}$such that system (9) is $\gamma$-stable.

In addition, the second inequality of (26) enables that the backward reachable set $\Psi(k, \mathcal{T})$ is contained in an ellipsoid $x(k)^{T} W x(k) \leq 1$ which is a contractive and invariant set. From Lemma 5, if there exists a control input $F(k-1)=$ $Y(k-1) W(k-1)^{-1}$ satisfying (26), one has $\Psi(k-1) \in \mathcal{R}$.

Furthermore, the last inequality of (26) holds on the condition that

$$
\underline{Y}_{i j} \leq Y_{i j} \leq \bar{Y}_{i j}
$$

Considering (27) and the interval arithmetic of addition and multiplication

$$
\begin{aligned}
a+b & =[\underline{a}+\underline{b}, \bar{a}+\bar{b}], \\
d \cdot a & =[\min (d \underline{a}, d \bar{a}), \max (d \underline{a}, d \bar{a})],
\end{aligned}
$$

for $a \in[\underline{a}, \bar{a}] \subset \mathbb{R}, b \in[\underline{b}, \bar{b}]$ and $d \in \mathbb{R}$, it further yields that the last inequality of (28) holds if $F(k)$ is constrained by a hyper-rectangle given by Remark 2 .

Finally, regarding the objective function of (26), we use the generalized Frobenius norm as our measure of a zonotope, i.e.,

$$
\begin{array}{ll} 
& \left\|\Psi_{k-1}\right\|_{F-W} \\
\stackrel{(11)}{=} & \left\|\mathcal{Z}\left(c_{k-1}, G_{k-1}\right)\right\|_{F-W} \\
= & \operatorname{trace}\left(\left(G_{k-1}^{T}\right)\left(W^{-1}\right)^{T}\left(W^{-1}\right)\left(G_{k-1}\right)\right) \\
\stackrel{(12)}{=} & \operatorname{trace}\left(G_{k}^{T}\left(\left(A+B Y W^{-1}\right)^{-1}\right)^{T}\left(W^{-1}\right)^{T} W^{-1}\right. \\
& \left.\cdot\left(A+B Y W^{-1}\right)^{-1} G_{k}\right) \\
= & \operatorname{trace}\left(G_{k}^{T}\left((A W+B Y)^{-1}\right)^{T}(A W+B Y)^{-1} G_{k}\right),
\end{array}
$$

from which one could observe that maximizing $\left\|\Psi_{k-1}\right\|_{F-W}$ can be approximated by minimizing trace $(R(W, Y))$. In other words, the backward reachable set $\Psi(k-1)$ is enlarged by the measure of the generalized Frobenius norm, which ends the proof.

\section{A NuMERICAL EXAMPLE}

The computation is carried out by using MATLAB 2015a on a standard laptop with an Intel Core i7-4712MQ processor and an 8GB DDR3 RAM. The MATLAB toolboxes CORA [35] and MPT3 [36] are used to compute the zonotopic and polyhedral sets, and toolboxes CVX, SDPSOL, SeDuMi, 
SDPT3, and LMITOOL are used for solving BMI and semidefinite problems (For various LMI solvers, see [34] and references therein).

We consider the following model:

$$
x(k+1)=(A+B F(k)) x(k)
$$

with

$$
A=\left[\begin{array}{ll}
0.8 & 0.1 \\
0.2 & 0.6
\end{array}\right], B=\left[\begin{array}{ll}
2 & 1 \\
1 & 1
\end{array}\right]
$$

and $\bar{F}_{11}=0.1, \underline{F}_{11}=0.5, \underline{F}_{12}=0.3, \bar{F}_{12}=0.5, \underline{F}_{21}=$ $0.2, \bar{F}_{21}=0.3, \underline{F}_{22}=0.3, \bar{F}_{22}=0.6$, where the origin $\mathrm{O}_{2}$ is a locally asymptotically stable equilibrium point with an unbounded RA. Let us denote the control strategy based on Theorem 1 as Strategy 1 and denote the control strategy based on Theorem 2 as Strategy 2.

First, we use Strategy 1 to compute the backward reachable sets and enlarge the estimated RA. we set the terminal set as $\mathcal{T}=\mathcal{Z}_{1}\left(c_{\mathrm{t} 1}, G_{\mathrm{t} 1}\right)$, where

$$
c_{\mathrm{t} 1}=\left[\begin{array}{l}
0 \\
0
\end{array}\right], G_{\mathrm{t} 1}=\left[\begin{array}{ll}
1 & 0 \\
0 & 1
\end{array}\right] \text {. }
$$

For each time step $k$, an optimal control input $F_{k}$ can be obtained by solving (22). Then, the backward reachable set $\Psi(k, \mathcal{T})$ can be computed from (13). The computation result by using this strategy is shown in Fig. 4. Let us consider a set $\Upsilon=\left\{x \in \mathbb{R}^{2}: 45 \leq x_{1} \leq 55,95 \leq x_{2} \leq 105\right\}$. Observe $\Upsilon$ is in the backward reachable set $\Psi\left(k_{\mathrm{t}}-4, \mathcal{T}\right)$, from Definition 1, we obtain the result that $\Upsilon$ can be steered into the target set $\mathcal{T}$ in 4 time steps by using the optimal control inputs $F\left(k_{\mathrm{t}}-i\right)$, for $i=4,3,2,1$.

Moreover, we can also apply Strategy 2 to compute the estimated RA. The terminal set is selected as $\mathcal{T}=$ $\mathcal{Z}_{2}\left(c_{\mathrm{t} 2}, G_{\mathrm{t} 2}\right)$, where

$$
c_{\mathrm{t} 2}=\left[\begin{array}{l}
1 \\
1
\end{array}\right], G_{\mathrm{t} 2}=\left[\begin{array}{ll}
2 & 0 \\
0 & 2
\end{array}\right] .
$$

The computation result is shown in Fig. 5. Note that different from Strategy 1, Strategy 2 does not require a set containment condition. In other words, the backward reachable set is not ensured to be an invariant set. See from Fig. 5 that $\Psi\left(k_{\mathrm{t}}-3\right)$ is not contained in $\Psi\left(k_{\mathrm{t}}-4\right)$.

TABLE III

THE COMPUTATIONAL TIME $t_{c}$ [sec] FOR DIFFERENT STRATEGIES, AND THE SELECTED BACKWARD TIME STEP $k_{\mathrm{b}}$.

\begin{tabular}{lccccc}
\hline & $k_{\mathrm{b}}=5$ & $k_{\mathrm{b}}=10$ & $k_{\mathrm{b}}=20$ & $k_{\mathrm{b}}=30$ & $k_{\mathrm{b}}=40$ \\
\hline Strategy 1 & 7.84 & 15.68 & 29.64 & 47.32 & 73.91 \\
Strategy 2 & 4.36 & 8.47 & 15.36 & 22.76 & 31.28 \\
\hline
\end{tabular}

The computational time of the proposed methods is given in Tab. III. Strategy 1 is not as efficient as Strategy 2; it is due to the fact that (22) needs to solve an optimization with constraints of BMIs, and BMI solver relies on some iterative algorithm (e.g., Gauss-Seidel method) to find the suboptimal solution [34].

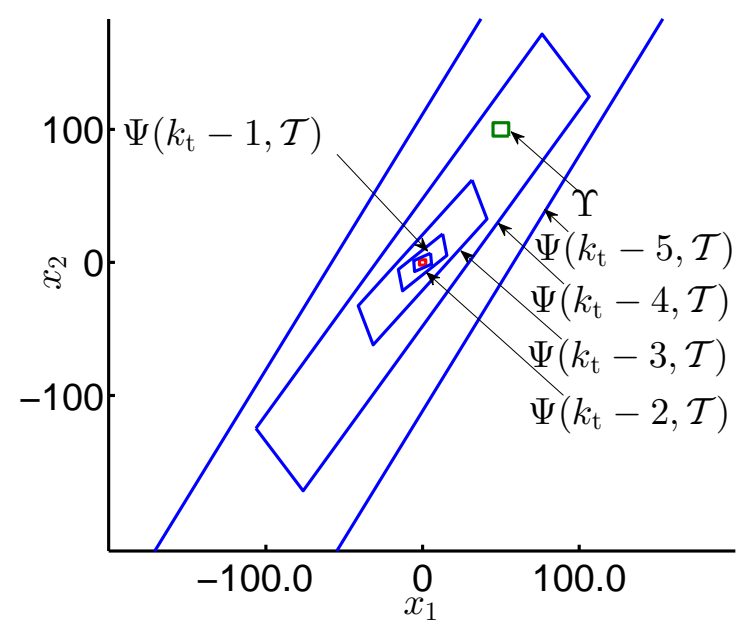

Fig. 4. Computation results by using Strategy 1.

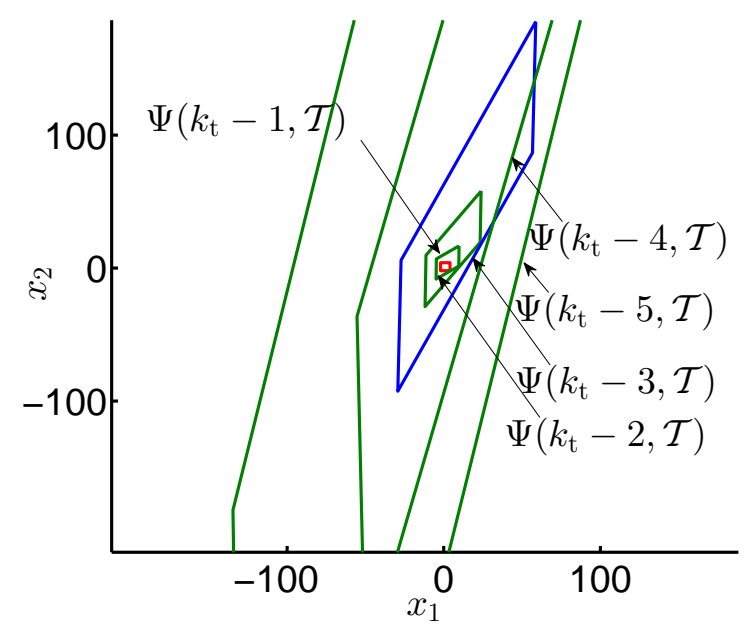

Fig. 5. Computation results by using Strategy 2 .

\section{Conclusion And Discussion}

An approach is proposed for estimating and enlarging the RA of linear discrete-time systems from a new perspective: computing the backward reachable sets. A Lagrangian method is used via zonotopic representation. Firstly, we propose a method for checking the zonotope containment via solving a Semi-Definite Programming (SDP). Based on this method, an optimal control input is obtained for enlarging the backward reachable set as the estimate of the RA by solving an optimization problem with Bilinear Matrix Inequalities (BMIs). Secondly, based on the Lyapunov stability theory, an optimal control strategy is proposed for each step via quadratic stabilization. The backward reachable set as the inner-approximation of the RA is maximized under the proposed control strategy by solving a convex optimization problem.

The main conservativeness of these approaches stems from the following two facts: 1) for the method based on 
zonotope containment, only a suboptimal solution can be obtained for the BMI problem (22), and the Frobenius norm of generator matrix is adopted for approximating the volume of zonotopes, which is non-convex with regards to the control variables [32]; 2) for the method based on quadratic stabilization, like other methods using Lyapunov stability theory, it also suffers from the fact that a Lyapunov function $x^{T} W x$ is required. In order to reduce the conservativeness, one promising convex approach is proposed by using the moment theory and the occupation measure, and conditions of LMIs are obtained other than BMI [12]. Another possible way is to construct a convex approach for enlarging the volume of zonotopes (see the analysis problem in [25]), to which our future effort will be devoted. Furthermore, we are also interested to extend this method to linearizable nonlinear systems, hybrid systems and large-scale cyberphysical systems [27], [37].

\section{ACKOWLEDGEMENTS}

Financial supports from the German Research Foundation (DFG AL 1185/2-1) and the Graduiertenkolleg 1480 (PUMA) are gratefully acknowledged.

\section{REFERENCES}

[1] C. Cosentino, L. Salerno, A. Passanti, A. Merola, D. G. Bates, and F. Amato, "Structural bistability of the GAL regulatory network and characterization of its domains of attraction," Journal of Computational Biology, vol. 19, no. 2, pp. 148-162, 2012.

[2] A. I. Doban and M. Lazar, "Domain of attraction computation for tumor dynamics," in Proceedings of the Conference on Decision and Control, 2014, pp. 6987-6992.

[3] M. L. Matthews and C. Williams, "Region of attraction estimation of biological continuous boolean models," in Proceedings of the International Conference on Systems, Man, and Cybernetics, 2012, pp. 1700-1705.

[4] V. I. Zubov and L. F. Boron, Methods of A. M. Lyapunov and their Application. Noordhoff Groningen, 1964.

[5] B. Tibken, "Estimation of the domain of attraction for polynomial systems via LMIs," in Proceedings of the Conference on Decision and Control, 2000, pp. 3860-3864.

[6] Z. Jarvis-Wloszek, R. Feeley, W. Tan, K. Sun, and A. Packard, "Some controls applications of sum of squares programming," in Proceedings of the Conference on Decision and Control, 2003, pp. 4676-4681.

[7] P. A. Parrilo, "Structured semidefinite programs and semialgebraic geometry methods in robustness and optimization," Ph.D. dissertation, California Institute of Technology, 2000.

[8] F. Amato, F. Calabrese, C. Cosentino, and A. Merola, "Stability analysis of nonlinear quadratic systems via polyhedral Lyapunov functions," in Proceedings of the American Control Conference, 2008, pp. 2291-2296.

[9] G. Chesi, "Rational Lyapunov functions for estimating and controlling the robust domain of attraction," Automatica, vol. 49, no. 4, pp. 10511057, 2013.

[10] A. Papachristodoulou and S. Prajna, "Analysis of non-polynomial systems using the sum of squares decomposition," in Positive polynomials in control, ser. Lecture Notes in Control and Information Science. Springer, 2005, vol. 312, pp. 23-43.

[11] W. Tan and A. Packard, "Stability region analysis using polynomial and composite polynomial Lyapunov functions and sum-of-squares programming," IEEE Transactions on Automatic Control, vol. 53, no. 2, pp. 565-570, 2008.

[12] D. Henrion and M. Korda, "Convex computation of the region of attraction of polynomial control systems," IEEE Transactions on Automatic Control, vol. 59, no. 2, pp. 297-312, 2014.

[13] I. M. Mitchell, A. M. Bayen, and C. J. Tomlin, "A time-dependent Hamilton-Jacobi formulation of reachable sets for continuous dynamic games," IEEE Transactions on Automatic Control, vol. 50, no. 7, pp. 947-957, 2005.
[14] J. N. Maidens, S. Kaynama, I. M. Mitchell, M. M. K. Oishi, and G. A. Dumont, "Lagrangian methods for approximating the viability kernel in high-dimensional systems," Automatica, vol. 49, no. 7, pp. 2017-2029, 2013.

[15] D. Panagou, K. Margellos, S. Summers, J. Lygeros, and K. J. Kyriakopoulos, "A viability approach for the stabilization of an underactuated underwater vehicle in the presence of current disturbances," in Proceedings of the Conference on Decision and Control, 2009, pp. 8612-8617.

[16] A. Chutinan and B. H. Krogh, "Verification of polyhedral-invariant hybrid automata using polygonal flow pipe approximations," in Proceedings of the International Conference on Hybrid Systems: Computation and Control, 1999, pp. 76-90.

[17] M. Althoff, O. Stursberg, and M. Buss, "Reachability analysis of nonlinear systems with uncertain parameters using conservative linearization," in Proceedings of the Conference on Decision and Control, 2008, pp. 4042-4048.

[18] A. Girard, "Reachability of uncertain linear systems using zonotopes," in Proceedings of the International Conference on Hybrid Systems: Computation and Control, 2005, pp. 291-305.

[19] A. B. Kurzhanski and P. Varaiya, "Dynamic optimization for reachability problems," Journal of Optimization Theory and Applications, vol. 108, no. 2, pp. 227-251, 2001.

[20] S. Kaynama, J. Maidens, M. Oishi, I. M. Mitchell, and G. A. Dumont, "Computing the viability kernel using maximal reachable sets," in Proceedings of the International Conference on Hybrid Systems: Computation and Control, 2012, pp. 55-64.

[21] D. Han and M. Althoff, "Control synthesis for non-polynomial systems: a domain of attraction perspective," in Proceedings of the Conference on Decision and Control, 2015, pp. 1160-1167.

[22] M. Althoff, "Reachability analysis and its application to the safety assessment of autonomous cars," Ph.D. dissertation, Technische Universität München, 2010.

[23] H. Kiendl, J. Adamy, and P. Stelzner, "Vector norms as Lyapunov functions for linear systems," IEEE Transactions on Automatic Control, vol. 37, no. 6, pp. 839-842, 1992.

[24] F. Blanchini, "Nonquadratic Lyapunov functions for robust control," Automatica, vol. 31, no. 3, pp. 451-461, 1995.

[25] T. Alamo, J. M. Bravo, and E. F. Camacho, "Guaranteed state estimation by zonotopes," Automatica, vol. 41, no. 6, pp. 1035-1043, 2005.

[26] V. T. H. Le, C. Stoica, T. Alamo, E. F. Camacho, and D. Dumur, "Zonotopic guaranteed state estimation for uncertain systems," Automatica, vol. 49, no. 11, pp. 3418-3424, 2013.

[27] M. Althoff and B. H. Krogh, "Reachability analysis of nonlinear differential-algebraic systems," IEEE Transactions on Automatic Control, vol. 59, no. 2, pp. 371-383, 2014.

[28] J. K. Scott, D. M. Raimondo, G. R. Marseglia, and R. D. Braatz, "Constrained zonotopes: A new tool for set-based estimation and fault detection," Automatica, vol. 69, pp. 126-136, 2016.

[29] R. A. Horn and C. R. Johnson, Matrix Analysis. Cambridge University Press, 1985.

[30] A. B. Kurzhanski and I. Vályi, Ellipsoidal calculus for estimation and control. Nelson Thornes, 1997.

[31] S. Boyd, L. El Ghaoui, E. Feron, and V. Balakrishnan, Linear matrix inequalities in system and control theory. Society for industrial and applied mathematics, 1994, vol. 15.

[32] E. Gover and N. Krikorian, "Determinants and the volumes of parallelotopes and zonotopes," Linear Algebra and Its Applications, vol. 433, no. 1, pp. 28-40, 2010.

[33] H. Fujioka and K. Hoshijima, "Bounds for the BMI eigenvalue problem," Journal of the Society of Instrument and Control Engineers, vol. 33, no. 7, pp. 616-621, 1997.

[34] J. G. VanAntwerp and R. D. Braatz, "A tutorial on linear and bilinear matrix inequalities," Journal of Process Control, vol. 10, no. 4, pp. 363-385, 2000.

[35] M. Althoff, "An introduction to CORA 2015," in Proceedings of the Workshop on Applied Verification for Continuous and Hybrid Systems, 2015.

[36] M. Herceg, M. Kvasnica, C. Jones, and M. Morari, "Multi-Parametric Toolbox 3.0," in Proceedings of the European Control Conference, 2013, pp. 502-510.

[37] M. Althoff, O. Stursberg, and M. Buss, "Computing reachable sets of hybrid systems using a combination of zonotopes and polytopes," Nonlinear analysis: hybrid systems, vol. 4, no. 2, pp. 233-249, 2010. 